Rev. Elev. Méd. vét. Pays trop., 1970, 23 (3) : 321-26

\title{
Le Prothidium et l'Isométamidium dans le traitement de la trypanosomiase du chien à Trypanosoma brucei
}

\author{
par S. M. TOURE \\ avec la collaboration technique de Y. KANE et N. BA
}

\begin{abstract}
RESUME
Le Prothidium et l'Isométamidium ont été utilisés dans le traitement de plusieurs cas de trypanosomiase expérimentale du chien à Trypanosoma brucei.

Le Prothidium est administré à raison de $2 \mathrm{mg} / \mathrm{kg}$ de la solution à 2 p. 100 et l'Isométamidium $1 \mathrm{mg} / \mathrm{kg}$ de la solution à $1 \mathrm{p} .100$. Tous deux sont injectés par voie intramusculaire profonde dans le creux poplité à des animaux présentant une forte parasitémie. Les trypanosomes disparaissent de la circulation généralement en deux ou trois jours. Les animaux tolèrent relativement bien le traitement.

Les deux médicaments, à leur posologie respective, protègent aussi dans une certaine mesure contre les réinfections expérimentales par inoculation de $T$. brucei.

L'apparition de chimiorésistance à l'égard du Prothidium a empêché, dans plusieurs cas, une protection effective.

Ces observations ont été faites dans des conditions artificielles et portent sur un nombre limité de cas. Elles ne prêtent pas à généralisation mais autorisent à penser que ces deux trypanocides pourraient être utilisés avec profit dans le traitement de la trypanosomiase du chien.
\end{abstract}

\section{INTRODUCTION}

Au cours de ces dernières années, le Laboratoire national de l'Elevage et de Recherches vétérinaires de Dakar a été plusieurs fois consulté pour le diagnostic et le traitement de cas de trypanosomiase canine contractée dans des régions du Sénégal infestées de glossines. Les chiens sont soit de race autochtone, soit d'une race de pays à climat tempéré. La maladie est due à Trypanosoma (Nannomonas) congolense BRODEN, 1904 ou bien à $T$. (Trypanozoon) brucei PLIMMER et BRADFORD, 1899. Il y a quelquefois infection mixte due aux deux espèces à la fois.

Alors que la maladie à $T$. congolense rétrocède rapidement et peut être guérie grâce à un seul traitement au Bérénil (solution à 7 p. 100 , $3,5 \mathrm{mg} / \mathrm{kg}$, voie intramusculaire), par contre la trypanosomiase à $T$. brucei est souvent difficile à soigner par les trypanocides classiques, y compris le Bérénil. Dans deux cas d'infection naturelle par $T$. brucei, le Bérénil a été administré à une dose double $(7 \mathrm{mg} / \mathrm{kg})$ mais le traitement a été suivi d'une rechute après plusieurs jours de guérison apparente. Il a été constaté aussi un cas de chimio-résistance absolue à l'égard du Bérénil, l'administration de ce médicament n'ayant eu aucun effet sur les trypanosomes. Cette absence d'efficacité s'explique par des traitements antérieurs au Bérénil. Faute de pouvoir traiter ces chiens par le Moranyl ou par la Lomidine pour des raisons de commodité (plusieurs interventions nécessaires) et même d'efficacité (chimiorésistance croisée), il nous a semblé opportun d'essayer d'autres trypanocides de plus grande activité : les dérivés plus récents du Phenanthridinium. 
Deux chiens, non infectés de trypanosomes ont reçu respectivement du Prothidium $(2 \mathrm{mg} / \mathrm{kg}$ de la solution à 2 p. 100 , voie intramusculaire profonde dans le creux poplité) et du chlorhydrate de chlorure d'Isométamidium (1 $\mathrm{mg} / \mathrm{kg}$ de la solution à 1 p. 100 , voie intramusculaire profonde). Ces chiens ont bien toléré ces médicaments et par la suite, le traitement a été appliqué avec succès dans des cas de trypanosomiase naturelle à $T$. brucei : deux interventions par le Prothidium et une par l'Isométamidium.

Pour avoir des données complémentaires sur le traitement par ces médicaments de la trypanosomiase canine à $T$. brucei, l'un et l'autre produit ont été administrés à des chiens d'expérience infectés par la seringue. Les observations qui suivent sont toutes relatives à la maladie expérimentale.

\section{METHODE}

Les chiens inoculés sont de race locale et ils ont été gardés intra muros dans un chenil. L'infection expérimentale est précédée par une étude de l'état général, plus particulièrement de la recherche de parasites autres que les trypanosomes. Trois des chiens sur seize observations hébergeaient dans leur sang des microfilaires de Dirofilaria ou de Dipetalonema.

Les animaux ont été ensuite infectés par une dilution de Trypanosoma brucei (souches d'origine canine) injectée par voie sous-cutanée. Le sang est examiné tous les jours et la température rectale relevée. La parasitémie est évaluée quantitativement par approximation:

(+) trypanosomes peu nombreux : un dans plusieurs champs de microscope et jusqu'à un par champ;

$(++)$ deux à plusieurs trypanosomes par champ, jusqu'à un nombre de dix;

$(+++)$ plusieurs trypanosomes par champ, de dix jusqu'à un grouillement parasitaire.

Lorsque la parasitémie est jugée importante $(++$ ou +++$)$, les chiens sont traités par le Prothidium ou par l'Isométamidium. Le Prothidium est administré en solution à 2 p. 100 à raison de $2 \mathrm{mg} / \mathrm{kg}$, par voie intramusculaire profonde dans le creux poplité. Quelques animaux ont reçu une dose double de Prothidium.
L'Isométamidium est injecté par voie intramusculaire profonde à raison de $1 \mathrm{mg} / \mathrm{kg}$ de la solution à 1 p. 100 . Dans quelques cas de rechute après traitement, le Bérénil a été administré à raison de $3,5 \mathrm{mg} / \mathrm{kg}$ ou $7 \mathrm{mg} / \mathrm{kg}$ de la solution à 7 p. 100 , voie intramusculaire dans les muscles lombaires.

La réinoculation de $T$. brucei aux animaux déjà traités permet de juger du pouvoir protecteur des trypanocides utilisés.

Le mode d'utilisation de ces médicaments, à l'exclusion de la dose administrée ne sera pas repris dans la relation des faits.

Les divers cas sont analysés individuellement dans les paragraphes qui suivent. Du fait des différences dans les résultats, cette méthode de présentation nous semble préférable à un exposé synthétique.

\section{Traitement par le Prothidium}

\section{Observation $n^{\circ} I$}

La parasitémie est apparente quatre jours après l'inoculation. Au $6^{\circ}$ jour de parasitémie, le chien est traité par le Prothidium, $2 \mathrm{mg} / \mathrm{kg}$. Les trypanosomes disparaissent totalement quatre jours après le traitement. Aucun trypanosome n'est observé pendant les dix jours suivants. Le chien est alors réinfecté mais il ne prend pas l'infection et reste sans trypanosomes pendant 34 jours. Il est réinfecté une seconde fois et il y a parasitémie au bout de cinq jours. Après dix jours de maladie, le chien est à nouveau traité par le Prothidium, $2 \mathrm{mg} / \mathrm{kg}$. Les trypanosomes disparaissent en quatre jours, mais quatre jours plus tard, soit huit jours après le traitement, il y a une rechute. L'animal est alors traité par le Bérénil, $7 \mathrm{mg} / \mathrm{kg}$. Les parasites disparaissent en 48 heures et aucun trypanosome n'a été observé pendant les 36 jours suivants.

\section{Observation $n^{\nu} 2$}

Parasitémie apparente trois jours après l'inoculation. Traitement par le Prothidium, au $7^{\text {e }}$ jour de parasitémie. Disparition totale des trypanosomes en trois jours. Douze jours après la guérison effective, le chien est réinfecté mais en 24 jours d'observation, aucun trypanosome n'a été décelé. Une deuxième réinfection est tentée au bout de ce temps mais elle reste sans effet : 43 jours sans trypanosomes. 


\section{Observation $n^{\circ} 3$}

Parasitémie huit jours après l'infection et d'emblée très forte. Traitement par le Prothidium, $2 \mathrm{mg} / \mathrm{kg}$. Disparition des trypanosomes deux jours après le traitement. Aucun trypanosome n'est observé pendant 21 jours. Au $22^{\circ}$ jour, il y a réapparition de trypanosomes. Présence de ceux-ci pendant douze jours consécutifs. L'animal est alors traité par le Bérénil, $3,5 \mathrm{mg} / \mathrm{kg}$. Les trypanosomes disparaissent en 24 heures. Sept jours après ce traitement, le chien reçoit à nouveau du Prothidium, $2 \mathrm{mg} / \mathrm{kg}$ puis est réinfecté au 7 jour. Aucun trypanosome n'est décelé pendant les 37 jours suivants.

\section{Observation $n^{0} 4$}

Parasitémie apparente trois jours après l'infection. Au $14^{*}$ jour de maladie, le chien est traité par le Prothidium, $2 \mathrm{mg} / \mathrm{kg}$. Disparition des trypanosomes au bout de deux jours. Pas de trypanosome observé pendant les 33 jours suivants, mais au $34^{\mathrm{e}}$ jour, réapparition de trypanosomes. Deuxième traitement au Prothidium, $2 \mathrm{mg} / \mathrm{kg}$. Disparition des trypanosomes en trois jours mais le $10^{\mathrm{e}}$ jour qui suit ce second traitement, il y a à nouveau rechute. Troisième traitement au Prothidium, $4 \mathrm{mg} / \mathrm{kg}$. Disparition des trypanosomes en deux jours mais nouvelle rechute 12 jours après ce troisième traitement. Le chien reçoit alors de l'Isométamidium, $1 \mathrm{mg} / \mathrm{kg}$. Observation négative deux jours après et absence de trypanosomes pendant les 19 jours suivants.

\section{Observation $n^{0} 5$}

Parasitémie apparente neuf jours après l'infection. Traitement au $17^{\mathrm{e}}$ jour de maladie par le Prothidium, $2 \mathrm{mg} / \mathrm{kg}$. Disparition des trypanosomes en trois jours et observations négatives pendant 28 jours. Au $29^{\mathrm{e}}$ jour, il y a rechute. Traitement par Prothidium $2 \mathrm{mg} / \mathrm{kg}$. Les trypanosomes disparaissent en trois jours mais nouvelle rechute six jours après le second traitement. Au $11^{\mathrm{e}}$ jour de la rechute, traitement par le Prothidium, $4 \mathrm{mg} / \mathrm{kg}$. Les trypanosomes disparaissent en trois jours et on n'en trouve pas pendant les 31 jours suivants.

\section{Observation $n^{\prime} 6$}

Parasitémie sept jours après l'infection. Au $13^{\mathrm{e}}$ jour de parasitémie, traitement par le Prothidium, $2 \mathrm{mg} / \mathrm{kg}$. Disparition des trypanosomes en deux jours et observations négatives les 34 jours suivants. Au $35^{\mathrm{e}}$ jour, réapparition des trypanosomes. Traitement par Prothidium, $4 \mathrm{mg} / \mathrm{kg}$. Négatif au bout de trois jours mais nouvelle multiplication des trypanosomes au $10^{\circ}$ jour qui suit le traitement. Le chien reçoit alors de l'Isométamidium, $1 \mathrm{mg} / \mathrm{kg}$. Les trypanosomes disparaissent en quatre jours. Observations négatives pendant les neufs jours suivants, mais l'animal meurt au bout de ce temps.

\section{Observation $n^{0} 7$}

Parasitémie six jours après l'infection. Au $15^{\mathrm{e}}$ jour de parasitémie traitement par Prothidium, $2 \mathrm{mg} / \mathrm{kg}$. Disparition des trypanosomes en quatre jours, mais rechute dix jours après ce traitement. Au $16^{\mathrm{e}}$ jour de la rechute, nouveau traitement par le Prothidium, $2 \mathrm{mg} / \mathrm{kg}$. Négatif au bout de deux jours mais réapparition des trypanosomes sept jours après. Traitement de la rechute par l'Isométamidium, $1 \mathrm{mg} / \mathrm{kg}$. Les trypanosomes disparaissent en deux jours et les observations sont négatives pendant treize jours, mais l'animal meurt au $14^{\mathrm{e}}$ jour.

\section{Observation $n^{\circ} 8$}

Parasitémie sept jours après l'infection. Au

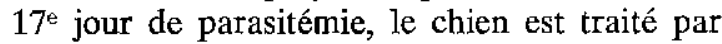
le Prothidium, $2 \mathrm{mg} / \mathrm{kg}$. Disparition des trypanosomes au bout de cinq jours, mais nouvelle parasitémie 48 heures après. Traitement par le Bérénil, $7 \mathrm{mg} / \mathrm{kg}$. Observations négatives pendant 38 jours.

Dans cette observation, les trypanosomes sont fortement chimiorésistants à l'égard du Prothidium. Au cours des expériences rapportées ci-dessous, relatives à l'Isométamidium, les chiens sont infestés par cette souche résistante.

\section{Traitement par l'Isométamidium}

\section{Observation $n^{\circ} 9$}

La souche infectante résiste au Prothidıum. Parasitémie apparente au $5^{\text {c }}$ jour qui suit l'infection. Au $4^{\text {e }}$ jour de parasitémie, le chien est traité par l'Isométamidium, $1 \mathrm{mg} / \mathrm{kg}$. Les trypanosomes disparaissent en trois jours et les observations sont négatives pendant les douze jours suivants.

\section{Observation $n^{\circ} 10$}

La souche infectante résiste au Prothidium. Parasitémie apparente au $6^{e}$ jour qui suit 
l'infection. Traitement par l'Isométamidium, $1 \mathrm{mg} / \mathrm{kg}$, au $4^{\mathrm{e}}$ jour de parasitémie. Disparition des trypanosomes en trois jours et observations négatives pendant douze jours.

\section{Observation $n^{\circ} 11$}

La souche infectante résiste au Prothidium. Parasitémie cinq jours après l'infection. Au $3^{\text {e }}$ jour de parasitémie, traitement par l'Isométamidium, $1 \mathrm{mg} / \mathrm{kg}$. Négatif en trois jours et au cours des 15 jours suivants.

\section{Observation $n^{\circ} 12$}

Les mêmes faits que la précédente.

\section{Observations $n^{0} 13,14,15$ et 16}

Ces expériences sont relatives au pouvoir protecteur de l'Isométamidium. Les quatre chiens ont reçu ce médicament avant l'infection, à raison de $1 \mathrm{mg} / \mathrm{kg}$. La réinfection est faite vingt jours après l'administration thérapeutique. Aucun trypanosome n'a été observé chez les quatre chiens au cours des trente jours suivants.

\section{DISCUSSION}

Dans ces essais de traitement, les doses adoptées pour le Prothidium et pour l'Isométamidium sont les mêmes que celles applicables aux ruminants. A cette posólogie, rien n'indique une mauvaise tolérance par les carnivores des trypanocides utilisés. La plupart des chiens ont reçu plusieurs fois l'un ou l'autre produit. Le Prothidium a même été administré dans plusieurs cas à raison de $4 \mathrm{mg} / \mathrm{kg}$. Pendant les 24 à 48 heures qui suivent le traitement, on note seulement de la somnolence et une diminution de l'appétit. Ce malaise ne persiste pas longtemps. Tous les animaux ont accusé un gain de poids notable entre le début et la fin des expériences.

Toutefois, bien que des effets secondaires graves n'aient pas été constatés, il importe d'émettre certaines réserves du fait des conditions artificielles de l'expérimentation. En particulier, on ne saurait rejeter a priori la possibilité de phénomène de photosensibilisation car les chiens ont toujours été à l'abri du soleil dans leur chenil.

Concernant les deux cas de décès relatés, il y a lieu de souligner que les deux animaux hébergeaient des microfilaires de Dirofilaria et qu'ils avaient été soumis en l'espace de trois mois à plusieurs traitements trypanocides équivalant pratiquement à un surdosage.

A dose normale, le Prothidium et l'Isométamidium ont l'un et l'autre un pouvoir trypanocide assez marqué à l'égard de $T$. brucei. Administrés à un moment où la parasitémie est forte $(+++)$, ils entraînent généralement au bout de trois jours la disparition totale des trypanosomes. Ce délai peut être ramené à deux jours voire à 24 heures quand la parasitémie est moins élevée. Il suffit normalement d'une seule intervention thérapeutique si les trypanosomes ne sont pas chimiorésistants.

Dans les observations ci-dessus, il y a manifestation d'effet trypanopréventif mais on ne saurait à partir d'elles préjuger valablement de la durée de protection conférée dans les conditions naturelles. La durée de protection peut être estimée à plus de 39 jours dans un cas (avec le Prothidium) et plus de 50 jours dans quatre autres (avec l'Isométamidium). L'imprécision quant à la valeur préventive de ces médicaments chez les chiens d'expérience tient essentiellement au fait que les réinfections successives par la seringue mettent en jeu un nombre relativement très élevé de trypanosomes, sans commune mesure avec la quantité inoculée par une glossine au cours de son repas.

Dans plusieurs des observations enfin, il y a eu réapparition de trypanosomes quelques jours à plusieurs semaines après le traitement au Prothidium. Ces cas de rechute pourraient provenir d'une acquisition progressive de chimiorésistance au Prothidium ou d'une réinfection aléatoire au cours des examens. L'Isométamidium s'est révélé très actif dans ces cas.

Les points essentiels qui se dégagent de l'expérimentation sont les suivants :

1. Le Prothidium, administré en solution à 2 p. 100 à raison de $2 \mathrm{mg} / \mathrm{kg}$, par voie intramusculaire profonde, a, chez le chien, une bonne activité curative à l'encontre de Trypanosoma brucei. La parasitémie est jugulée en trois jours et le chien semble bien tolérer le traitement. Mais la rechute n'est pas exclue si l'animal avait déjà reçu ce médicament.

2. L'Isométamidium, en solution à 1 p. 100 , à la dose de $1 \mathrm{mg} / \mathrm{kg}$, par voie intramuscu- 
laire profonde, a, chez le chien, une bonne activité trypanocide sur $T$. brucei. La parasitémie est vaincue en deux ou trois jours et l'animal semble bien tolérer le traitement.

3. Le Prothidium et l'Isométamidium à leur posologie respective ont chacun un pouvoir trypanopréventif contre la trypanosomiase du chien à $T$. brucei. Sauf résistance avérée, la durée de protection est de plusieurs semaines mais cette durée pourrait, dans les conditions naturelles, être de plusieurs mois comme chez les Herbivores. Elle est en tout cas très limitée quand la souche infectante devient chimiorésistante.

4. Dans les infections rebelles, le Prothidium, à raison de $4 \mathrm{mg} / \mathrm{kg}$, peut enrayer l'infection si les trypanosomes ne manifestent pas une chimiorésistance marquée. Cette dose double ne semble pas, par elle-même, toxique pour le chien.

Le Bérénil a permis de lutter contre la résistance au Prothidium de $T$. brucei.

5. L'Isométamidium enfin, à raison de $1 \mathrm{mg} / \mathrm{kg}$ est à même d'agir sur $T$. brucei résistant au Prothidium aussi efficacement que le Bérénil.

Ces observations, quoiqu'en nombre assez limité, autorisent à penser eu égard à l'expérience acquise chez les Herbivores, ruminants et monogastriques, que les deux médicaments peuvent être utilisés dans le traitement de la trypanosomiase du chien.

\footnotetext{
Institut d'Elevage et de Médecine vétérinaire des Pays tropicaux. Laboratoire national de l'Elevage et de Recherches vétérinaires. Dakar-Hann.
}

\section{SUMMARY}

Prothidium and Isometamidium as drugs against Trypanosoma brucei canine trypanosomiasis

In some cases of experimental dog trypanosomiasis by Trypanosoma brucei inoculation, treatment was carried with Prothidium and Isometamidium. Prothidium was administered as 2 per 100 solution at $2 \mathrm{mg} / \mathrm{kg}$ and Isometamidium as 1 per 100 solution at $1 \mathrm{mg} / \mathrm{kg}$. Both were injected by deep intramuscular route into popliteus muscle. Treatment was done when parasitaemı at a high level and in most of these cases trypanosomes disappeared from the blood whithin the 2 or 3 days following the medication. Dogs did not suffer from drug administration.

These two trypanocidal drugs, at their respective dose, prevented animals from acquiring subsequent $T$. brucei experimental infection.

When in presence of induced resistance against Prothidium, it seemed difficult to obtain effective protection.

Because of the restricted number of related observations and of the artificial management of experiments, the results are not to be generalized but it is hoped that successful use could be made of these trypanocidal drugs in dog trypanosomiasis treatment.

\section{RESUMEN}

EI Prothidium y el Isometamidium en el tratamiento de la tripanosomiasis del perro con Trypanosoma brucei

Se utilizaron el Prothidium y el Isometamidium en el tratamiento de varios casos de tripanosomiasis experimental del perro con Trypanosoma briscei.

Se administra el Prothidium en dosis de $2 \mathrm{mg} / \mathrm{kg}$ de la solución a 2 p. 100 y el Isometamıdum en la de $1 \mathrm{mg} / \mathrm{kg}$ de la solución a $1 \mathrm{p} .100$. $\mathrm{Se}$ inyectan los dos medicamentos por via intramuscular honda en el hueco poplíteo en dos animales presentando una importante parasitemia. Los tripanosomos desaparecen generalmente a los dos o tres dias. Los animales toleran relativamente bien el tratamiento. Los dos medicamentos, en su posologia respectiva, protegen tambien contra las nuevas infestaciones experimentales por inoculación de $T$. brucei.

La aparición de quimioresistancia para con el Prothidium impidió, en varios casos, una protección efectiva. Se hicieron estas observaciones en condiciones artificiales; conciernen un número limitado de casos. No se puede generalizarlas pero permiten pensar que se podria utilizar dichos dos tripanocidos con exito en el tratamiento de la tripanosomiasis del perro. 


\section{BIBLIOGRAPHIE}

BERG (S.S.), BROWN (K.N.), LUCAS (J.M S.), « The trypanocidal activity and local tolerance of sparingly soluble salts of Metamidium », Anr. Trop. Med. Parasit., 1961, 55, 3, 298-304.

FINELLE (P.), LACOTTE (R.), « Action trypanocide de deux sels d'Isométamidium ", Rev. Elev'. Méd. vét. Pays trop., 1963, 16, 4, 405-409.

FINELLE (P.), LACOTTE (R.), "Essais de médicaments trypanopréventifs chez les ânes», Comité Scient. Intern. Rech. Trypano. 10 Réunion, Kampala 1964, CCTA п' 97, pp. 31-33.
HOARE (C.A.), «Morphological and taxonomic studies on mammalian trypanosomes. X. Revision of systematics », J. Protozool., 1964, 11, 2, 200207.

STEEL (E.D.), «Expériences au moyen de Samorin (isochlorure de Métamidium) pour la chimiothérapie et la chimioprophylaxie des infections par Trypanosoma simiae chez les porcs 》 (original en anglais). Comité Scient. Intern Rech. Trypano. 11e Réunion, Nairobi 1966, ISCTR (66) 28. 